SCIENCE'S COMPASS

ticular potential origins a competitive advantage over the vast excess of alternative potential origins in the same chromosome.

How could specific sequences give nearby potential origins an advantage over more distant potential origins? Several possibilities come to mind. First, certain sequences may induce a local chromatin structure that promotes origin function, perhaps by facilitating access to initiation factors. Second, certain sequences may specify localization within a portion of the nucleus that is favorable for origin function. Third, specific sequences may favor neighboring over distant potential origins by permitting earlier replication of the nearby origins. Earlier origins can suppress neighboring potential (but later) origins, because forks from the earlier origins replicate the later ones before they have a chance to initiate replication. Perhaps the reason for little origin specificity in small plasmids is that their miniature dimensions do not permit the necessary internal differences in chromatin structure, nuclear location, or replication timing.

Thus, the report by Aladjem et al. (1) provides solid evidence for specific replication origins determined by specific DNA sequences (replicators) in adult animal cells. It describes a genetic technique that should permit fine mapping of replicators. Future analyses of replicators and of the proteins that bind to them are likely to shed light on such still mysterious subjects as the mechanism of initiation of DNA replication, the roles of chromatin structure and nuclear location in initiation, and the molecular mechanisms that determine replication timing.
References and Notes

1. M. I. Aladjem, L. W. Rodewald, J. L. Kolman, G. M. Wahl, Science 281, 1005 (1998).

2. A.-K. Bielinsky and S. A. Gerbi, ibid. 279, 95 (1998)

3. D. Kitsberg, S. Selig, I. Keshet, H. Cedar, Nature 366, 588 (1993).

4. M. I. Aladjem et al., Science $\mathbf{2 7 0} 815$ (1995).

5. W. C. Forrester et al., Genes Dev. 4, 1637 (1990).

6. A. Dutta and S. P. Bell, Annu. Rev. Cell Dev. Biol. 13, 293 (1997).

7. C. S. Newlon, in DNA Replication in Eukaryotic Cells, M. L. DePamphilis, Ed. (Cold Spring Harbor Laboratory, Plainview, New York, 1996), pp. 873-914.

8. M. L. DePamphilis, Annu. Rev. Biochem. 62, 29 (1993).

9. P. J. Krysan and M. P. Calos, Mol. Cell. Biol. 11, 1464 (1991); S. S. Heinzel, P. J. Krysan, C. T. Tran, M. P. Calos, ibid. 11, 2263 (1991).

10. D. Coverley and R. A. Laskey, Annu. Rev. Biochem. 63 , 745 (1994).

11. J.-R. Wu, S. M. Keezer, D. M. Gilbert, EMBO J. 17, 1810 (1998).

12. I thank W. Burhans, S.-M. Kim, D. Kowalski, D. Mahoney, M. Weinberger, and J. Yates for valuable comments on the manuscript.

\section{Global Climate Data and Models: A Reconciliation}

\author{
James E. Hansen, Makiko Sato, Reto Ruedy, Andrew Lacis, Jay Glascoe
}

T he global warming issue has been confounded for several years by satellite observations of a cooling trend in the troposphere (the lowest 10 to $15 \mathrm{~km}$ of the atmosphere) (1-4). Such cooling is at odds with measurements at meteorological stations $(5,6)$, which show warming at Earth's surface, and with climate models, which yield warming at the surface and in the troposphere $(1,7)$.

Wentz and Schabel ( 8 ) have now discovered an oversight in the satellite data analysis that, at least to first order, removes the inconsistency between surface and satellite observations. The modified temperature profile is also in close accord with published climate model simulations. We caution that there are remaining uncertainties in the data, the models, and the analyses that are likely to prolong the debate about temperature trends. But the apparent reconciliation, if it stands up, can potentially lead to improved understanding of how sensitive the climate system is to natural and anthropogenic forcings such as changes of solar irradiance, volcanic eruptions, and greenhouse gases.

The oversight was failure to account for decay of satellite altitude caused by atmospheric drag. The Microwave Sounding Unit (MSU) on polar-orbiting weather

The authors are at the Goddard Institute for Space Studies, NASA, New York, NY 10025, USA. E-mail: jhansen@giss.nasa.gov satellites scans across the subsatellite orbital track, making measurements at discrete angles relative to the nadir. But as the satellite falls, the measurements at a specific "look" angle (relative to the nadir) have a changing angle relative to Earth's surface. This angle change has little or no effect on temperatures measured for the stratosphere (labeled channel S) and middle troposphere (channel MT), because they are inferred from near-nadir measurements (2). However, the lower tropospheric temperature (channel LT) is obtained from measurements made at several look angles, and thus it must be corrected for satellite altitude change. Altitude decay is most rapid, and thus the correction is largest, near the times of peak solar activity that occurred around 1980 and 1990. Wentz and Schabel do not reanalyze the MSU data from scratch, but they calculate the mean correction for 1979 to 1995 to be $+0.12^{\circ} \mathrm{C}$ per decade (see the figure).

The open triangle near the bottom of the figure is the global mean MSU LT temperature trend for 1979 to 1995 without correction for orbital decay. The solid triangles, connected by the solid line, are the MSU data at all three levels, LT, MT, and $\mathrm{S}$, including the correction of LT calculated by Wentz and Schabel. The triangle at 1000 mbar is the surface temperature change based on surface air measurements over land and sea surface temperature measurements over ocean $(5,6)$.
The correction of Wentz and Schabel makes the MSU temperature profile more self-consistent. Before the correction, MT was warming relative to LT, which presented an enigma. Each of the MSU measurements refers to a large layer of atmosphere, as indicated by the "weighting functions" on the right side of the figure. The broad weighting function for MT includes a contribution of about $15 \%$ from the lower stratosphere, a region that is known to have cooled rapidly in this period, on the basis of radiosonde, MSU $\mathrm{S}$, and other measurements $(1-3)$. The correction of Wentz and Schabel removes the enigma, as it leaves MT cooling relative to LT.

The observed temperature change is compared with our published climate model simulations in the figure. The climate model (7) was driven by observed SSTs and measured climate forcings added cumulatively one by one, as indicated in the figure. The climate forcings, which are imposed changes of Earth's radiation balance with space, are those measured during the era of satellite data, specifically stratospheric aerosols from volcanic eruptions, increasing greenhouse gases, changes of solar irradiance, and ozone depletion. An ensemble of five simulations, illustrated individually elsewhere (7), was made for each set of forcings, the individual runs differing because of small perturbations of initial atmospheric conditions and the chaotic nature of the atmosphere. Circles in the figure are the five-run means after weighting the calculated temperature profiles with the MSU weighting functions (2). Standard deviations among the five runs are about $0.02^{\circ} \mathrm{C}$ at each of the four levels shown in the figure. 


\section{SCIENCE'S COMPASS}

The model driven by all these measured climate forcings is in good agreement with the corrected observations, with warming in the troposphere and cooling in the stratosphere. The model atmosphere warms slightly more than observations, but the difference is within uncertainties caused by inaccuracies in the climate forcings, in the climate model, and in the temperature observations. The surface temperature change in these model runs is constrained by the use of observed SSTs, but the change of temperature profile is qualitatively similar when the model calculates SST (7). Ozone depletion is the main forcing that causes cooling at the higher atmospheric levels (7, 9). The agreement between the observed temperature profile and the independently obtained model profile lends credence to both the Wentz and Schabel correction and the model results.

If the corrected MSU profile is confirmed to be accurate, it will open the possibility of exploiting more fully the richness of the globally comprehensive MSU data for precise analyses of climate sensitivity to known climate forcings. Discrimination among different forcings is feasible because of their distinct spatial and temporal distributions. Although chaotic (unforced) atmospheric variability complicates interpretations (7), this difficulty will diminish as the satellite record lengthens.

Our opinion is that Wentz and Schabel have identified the primary reason for inconsistencies between MSU LT and other temperature data, including the other MSU channels, and that the need to correct MSU LT temperature for decaying satellite altitude cannot be denied. It is unlikely, however, that the exact proposed correction will be accepted without challenge and possible revision. This is because the analysis used to connect MSU measurements from successive satellites and correct for changes of daily observation time (2) are intricate and labyrinthine, and it may be argued that these procedures inadvertently partially compensate for the orbital decay effect.

It is desirable to have several independent documented analyses of the raw MSU data that avoid effects of orbit decay. MSU was designed for weather applications, not precise long-term climate monitoring, and thus it is difficult to achieve high accuracy. Christy et al. (2) work with temperature anomalies relative to a specific satellite, using periods of overlapping data to concatenate successive satellites. The method is complicated by satellite diurnal drift that causes measurements to be taken at a changing time of day, although temperatures are known to vary diurnally. Although the analysis attempts to account for such effects, it results in complex algorithms and arbitrary error specification.

Some confirmation of our expectation that the MSU analyses will change fur-

ing trend of $+0.11^{\circ} \mathrm{C}$ per decade, which is stronger than that found by Christy et $a l$., with the difference occurring mainly in 1991 when the NOAA 12 satellite succeeded NOAA 11. The concatenation of these satellites was identified in independent studies $(12,13)$ as a possible flaw in the Christy et al. analysis of LT temperatures. Thus, it is possible that the profile of MSU observations in the figure may shift toward greater warming. But the calibrations used by Prabhakara et al. are not immune to the effects of satellite diurnal drift that plague Christy et al. The analysis of Prabhakara et al. must be documented and scrutinized to assess its reliability.

An independent measure of tropospheric temperature is provided by radiosondes (weather balloons). Analyses for the Northern Hemisphere, where the sonde coverage is good, range from a tropospheric temperature trend near zero $(l)$ to about $+0.1^{\circ} \mathrm{C}$ per decade (7). Despite problems with radiosondes, especially changes of instrumentation, the radiosonde data suggest that any tropospheric warming trend cannot be much greater than that indicated by the MSU data as corrected by Wentz and Schabel.

Radiosondes, in fact, have played a larger role in this debate than appears on the surface. The accuracy of $0.03^{\circ} \mathrm{C}$ claimed for MSU (2) has as much to do with perceived agreement with radiosonde data as with the inherent accuracy of the satellite data, as evidenced

ther is provided by J. Christy (10), who reports that additional corrections may be needed for changes in instrument exposure to sunlight during satellite diurnal drift. He expects this correction to cause a cooling of the derived temperatures at the MSU MT level but to have little effect on MSU LT.

On the other hand, the MSU data at the MT level are being pushed in the opposite direction by an independent analysis of the same observations (11). This alternative approach by Prabhakara et al. (11) relies more on MSU prelaunch calibrations. This analysis uses only nadir views and is restricted to channel MT temperatures averaged over all land and over all ocean. The method yields a warm- by discovery of the $0.12^{\circ} \mathrm{C}$ MSU correction $(8)$. But radiosondes suffer from their own limitations, especially limited and declining spatial coverage, undocumented changes in the instruments, and inadequate calibration. If these problems were addressed, in the future radiosondes could not only help define long term climate change, but could also provide information with the high vertical resolution needed for analyzing the mechanisms causing climate change. An adequate observational system requires the combination of globally uniform satellite data and the vertical resolution and absolute calibration of an improved radiosonde system.

Climate model simulations agree well with the corrected MSU data, but these re- 


\section{SCIENCE'S COMPASS}

sults may change as the input climate forcings and the model itself are improved. One uncertain forcing is ozone change: Recent satellite data (14) suggest that the tropopause ozone depletion used in the figure was too great, implying that upper level cooling may be reduced with better ozone data. Forcing by anthropogenic aerosols, which may cause tropospheric cooling, is excluded for want of aerosol data. The most relevant deficiency of the model itself is probably its poor vertical resolution, with only one to two layers in the stratosphere: This is believed to reduce the model's stratospheric response to a climate forcing.

Despite the caveats that must accompany both the data and models, the convergence of satellite data, surface data, and models is an important step toward improved understanding of climate change. We expect the correction of Wentz and
Schabel to survive scrutiny, at least to first order, and that the resulting temperature distributions will lead to better understanding of climate sensitivity to forcings. In crediting Wentz and Schabel for discovering the satellite altitude effect, we should not forget the credit that Christy and Spencer deserve for pioneering MSU analysis and bringing it to the point that a correction of $0.1^{\circ} \mathrm{C}$ has such a large effect on interpretations of climate change.

The bottom line of this convergence could be a sea change in the global warming debate. Until now, the MSU data have been the principal refuge for those who deny the reality of global warming. We believe that warming trends of both the surface and troposphere are now sufficiently clear that the issue should no longer be whether global warming is occurring, but what is the rate of warming, what is its practical significance, and what should be done about it.

\section{References and Notes}

1. J. T. Houghton et al., Eds., Climate Change 1995 Cambridge Univ. Press, Cambridge, 1996).

. J. R. Christy, R. W. Spencer, R. T. McNider, J. Clim. 8 888 (1995).

3. J. Hansen et al., Clim. Change 30, 103 (1995).

4. J. Hurrell and K. Trenberth, Nature 386, 164 (1997)

5. J. Hansen, R. Ruedy, M. Sato, R. Reynolds, Geophys. Res. Lett. 23, 1665 (1996).

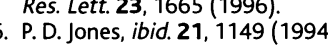

6. P. D. Jones, ibid. 21, 1149 (1994).

7. J. Hansen et al., J. Geophys. Res. 102, 25679 (1997)

8. F. J. Wentz and M. Schabel, Nature 394, 661 (1998).

9. C. K. Folland et al., Geophys. Res. Lett. 25, 353 (1998).

10. J. Christy, unpublished data.

11. C. Prabhakara, R. lacovazzi, J. M. Yoo, G. Dalu, Geophys. Res. Lett. 25, 1927 (1998)

12. P. D. Jones, T. J. Osborn, T. M. Wigley, P. M. Kelly, B. J. Santer, J. Geophys. Res. 102, 30135 (1997).

13. J. W. Hurrell and K. E. Trenberth, J. Clim. 11, 945 (1998).

14. H. J. Wang, D. M. Cunnold, X. J. Bao, J. Geophys. Res. 101, 12495 (1996)

15. We thank J. Christy, M. Schabel, F. Wentz, and D. Gaffen for discusssions.

\section{Protostars and Planets}

\section{Sara S. Russell and Alan P. Boss}

$\Delta$ the start of the century, planets and comets were nuisances that fogged the plates of astronomers and interfered with the study of stars. As the century draws to a close, the question of the origin of planets and their host stars is increasingly a quest of astronomers. Since 1995, when Geneva Observatory astronomers Michel Mayor and Didier Queloz found that the star 51 Pegasi wobbled because of a companion with a mass half that of Jupiter, the pace of Enhanced online at discoveries and shifts in www.sciencemag.org thinking on this subject has been swift. At a recent conference ( 1 ) in Santa Barbara, California, astronomers, planetary scientists, and meteoriticists met to review progress made on star and planet formation in the last decade. Improved observational tools have provided many exciting new discoveries (see figure), including our first peeks at other planetary systems, both mature and in the making.

One recent observational finding has been proof of the existence of the Kuiper Belt (2), a swarm of icy minor planets at the edge of our solar system, at a distance of about 30 to $50 \mathrm{AU}(1 \mathrm{AU}=$ distance between Earth and the sun) from the sun. Since the first Kuiper Belt object was dis-

S. S. Russell is at the Natural History Museum Cromwell Road London, SW7 5BD UK E-mail: sarr@nhm.ac.uk. A. P. Boss is at the Department of Terrestrial Magnetism, Carnegie Institution of Washington, Washington, DC 20015-1305. E-mail: boss@dtm.ciw.edu covered in 1992, astronomers have found about 70 such objects. The mass of the Kuiper Belt is now estimated to be about a tenth of the mass of Earth, which is only $1 \%$ of the mass inferred to have been present in the early outer solar system. At the meeting, David Jewitt (University of Hawaii) explained that the reason for mass loss in this region is unclear but may be due to collisional grinding of the bodies, producing dust that is subsequently lost.

This explanation for the Kuiper Belt's paucity of mass agrees well with observations of dust belts around other stars such as Beta Pictoris, where the dust is thought to come from collisions between more massive, unseen bodies. Jane Greaves (Joint Astronomy Centre, Hawaii) and co-workers reported imaging dust emission around the nearby star Epsilon Eridani, a star only slightly less massive than the sun. Most of the dust lies between 35 and $75 \mathrm{AU}$ from the star and has a combined mass of between 0.01 and 0.4 times the mass of Earth. Inhomogeneities in the distribution of dust may point to the possible presence of planets in this system, perhaps including a giant planet with an orbit at least as large as that of Neptune. Epsilon Eridani is between 0.5 and 1 billion years old, less than a quarter of the age of the sun, and it may provide an early analog to our own solar system.

Luis Rodriguez (Universidad Nacional Autonoma de Mexico, Mexico) and colleagues have used the Very Large Array of radiotelescopes to image much younger circumstellar disks with unprecedented spatial resolution. Rodriguez was able to image disks with masses of 0.03 and 0.06 solar masses around the binary protostars at the center of L1551 IRS5, the prototypical bipolar outflow. These disks, with radii of only about $10 \mathrm{AU}$, may be massive enough to be capable of forming giant planets rapidly, in which case a giant planet that forms near the edge of one of the disks is likely to be ejected outward because of gravitational tugs from the other protostar. The L1551 IRS5 system might then be an example of the type of system presumed necessary to account for the possible ejection of a giant planet (TMR1C) from a similarly sized and extremely youthful binary protostar system, reported by Susan Terebey (Extrasolar Research Corporation) and colleagues.

Two new extrasolar planets were discussed at the meeting, bringing the total number of known extrasolar planets to about a dozen. One, found around the star 14 Herculis, was discovered by Mayor and co-workers. This planet has the longest orbital period (4.4 years) of the planets discovered by the radial velocity method. The second planet orbits Gliese 876, the lowest mass star ( $1 / 5$ of the sun's mass) so far discovered to have a planet. Gliese 876 's planet was found independently by groups led by Xavier Delfosse (Geneva Observatory) and Geoffrey Marcy (San Francisco State University). Interestingly, there is a strong correlation between the metallicity of these stars (the abundance of elements heavier than helium) and the presence of orbiting planets: Essentially all of the stars with planets are metal-rich.

Brown dwarf stars, too low in mass to undergo sustained nuclear fusion, appear to orbit around less than $1 \%$ of solar-type 